\title{
A new generic class of Frankl's families
}

\author{
Pierre Colomb $^{1 \star}$, Alexis Irlande ${ }^{2 \star \star}$, Olivier Raynaud ${ }^{1 \star \star \star}$, Yoan Renaud $^{1 \dagger}$ \\ 1 Université Blaise Pascal, Aubirè, France \\ 2 Universidad Nacional de Colombia, Bogotá, Colombia
}

FECHA DE RECEPCIÓN: 5 DE ENERO DE 2011

FECHA DE APROBACIÓN: 22 DE ENERO DE 2011

\begin{abstract}
Frankl's conjecture states that in a family of sets closed by union $\mathcal{F}$ such that $\mathcal{F} \neq\{\emptyset\}$, there is an element that belongs to at least half of the sets of $\mathcal{F}$. There are several partial results of this conjecture. For example, it has been shown that families in which the smallest set is of size 1 or 2 , or families closed both by union and by intersection are Frankl's. In this article, by basing ourselves on an unseen recursive definition of the family of sets closed by union, we will define a new class of Frankl's families. Subsequently, we will evaluate the size of this class for the first 6 values of $n$. Finally we will show that this class does not coincide with the already known Frankl's classes.
\end{abstract}

Resumen. La conjetura de Frankl propone que en una familia de conjuntos cerrados bajo unión $\mathcal{F}$ tal que $\mathcal{F} \neq\{\emptyset\}$, existe un elemento que pertenece al menos a la mitad de los conjuntos de $\mathcal{F}$. Hay varios resultados parciales acerca de esta conjetura. Por ejemplo, se ha demostrado que las familias en las que el conjunto más pequeño es de tamaño 1 o 2 , o familias cerradas bajo unión y la intersección son de Frankl. En este artículo, basándonos en una definición recursiva nueva de la familia de conjuntos cerrados bajo unión, se definirá una nueva clase de familias de Frankl. Posteriormente se evaluará el tamaño de esta clase para los primeros 6 valores de $n$. Por último se demostrará que esta clase no coincide con las clases de Frankl, ya conocidas.

Keywords: combinatorics, Frankl's family, set, conjecture.

Palabras Clave: combinatoria, familia de Frankl, conjunto, conjetura.

\footnotetext{
* Doctor en Informática, Universidad de Clermont Ferrand, Francia. colomb@isima.fr

** Doctor en Informática, Universidad de Evry, Francia. irlande@lirmm.fr

$\star \star \star$ Doctor en Informática, Universidad de Montpellier, Francia. raynaud@isima.fr

$\dagger$ Doctor en Informática, Universidad de Clermont Ferrand, Francia. yoan.renaud@gmail.com
} 


\section{Introduction}

Frankl's conjecture [1, also called the conjecture of union-closed sets, is one of the most well-known open combinatorial problems. In its present form, this conjecture tells us that in a family of union-closed sets $\mathcal{F}$ such that $\mathcal{F} \neq\{\emptyset\}$, there is an element that belongs to at least half of the sets of $\mathcal{F}$. In literature, families verifying this property are often referred to as "Frankl's families" and the reference items, present in more than half of the sets, are called pivot. As of this day, there are a large number of partial results for this conjecture. These results are heterogeneous in nature.

One of the initial memorable results dates back to 1989 (cf. [2]), wherein the authors showed that the families containing at least one singleton or a 2-element set are Frankl's families. Thus, a pivot is the item or one of the items of this smaller set. However, this intuition that a smaller set could contain a pivot was invalidated in the same article by giving an example of family whose smallest set of size 3 did not contain the pivot.

When this conjecture was formulated, we find the hungarian mathematician Peter Frankl who, in the 70s, stated this surprisingly simple intuition that an intersection-closed family includes at least one item absent from half of the sets. We will find a reference to these works in [3. This initial version of the conjecture henceforth took the name of "Frankl's". This "intersection" version is specifically adapted to the lattice theory and has therefore be studied in this context ([4]) and quiet independently. One had to wait for the end of the century and works of Abe ([5]) to formally link the two versions.

The lattice approach of this problem allowed determining several classes for which the conjecture is true. Thus in 1992, Poonen determined in [6] that the conjecture is verified for lattice $\mathcal{L}$ such that for each $X$ belonging to $\mathcal{L}$, the interval $[0, X]$ is complemented, given that this class is a superset of the geometric lattice class. In 1998, Abe also showed ([7]) that Frankl's conjecture is verified for the modular lattices and extended this result to some cases of sub-modular lattices [5] . It was also shown that any distributive lattice, corresponding to an intersection-closed and union-closed family, verifies the conjecture. A complementary approach to the one above was to study local specificities to a family and to show how these specificities could allow reaching a conclusion about the presence or absence of a pivot. Thus, still in [6], Poonen defined a weight function for the sub-structures of a family and showed that if the weight is sufficient then the family containing this structure is Frankl's. This powerful theorem relies on a major result in topology. More recently, in 2008, a simplified version of this theorem was stated in [9] and used to show that any family on the univers $\{0, \ldots, 10\}$ is a Frankl's family. Lastly, other partial and interesting results are given in 10111213 .

The approach of the conjecture proposed in this article is innovative and relies on the recursive definition of the set of union-closed families. While this decomposition result does not yet as of date allow us to demonstrate by structural induction that the Frankl's conjecture is true for any family, it nevertheless allows us to define a very large class of families for which the Frankl's intuition is true. 
We will specifically show that this new class is transversal to most of classes already demonstrated to be Frankl's.

The balance of this article is structured as follows. The second section broadly reviews the recursive decomposition theorem of the set of union-closed families. In the third section, we present our new class of families verifying the conjecture and evaluating the size of this class for the initial values of $n$. Lastly, by giving some counterexamples, we will show that our class does not coincide with some specific classes known to be Frankl's. The conclusion summarizes the work done and the perspectives.

For clarity, we note elements by numbers $(1,2,3, \ldots)$. Sets are denoted by capital letters $(A, B, C, \ldots)$. Families of sets are denoted by cursive letters $(\mathcal{A}, \mathcal{B}$, $\mathcal{C}, \ldots)$. Finally, we note the sets of families of sets by black board letters $(\mathbb{A}, \mathbb{B}$, $\mathbb{C}, \ldots)$.

\section{Recursive decomposition of the set of union-closed families}

In the following we will note $\mathbb{M}_{n}$ the set of families on a universe $U_{n}=\{0, \ldots, n-1\}$, closed by union and containing the empty set. A union-closed family $\mathcal{M}$ on $U_{n}$ can be decomposed into two parts. The part consisting of the sets of $\mathcal{M}$ containing the element $n-1$ (denoted by $\mathcal{M}_{\text {sup }}$ for the upper part), and the complementary part (denoted by $\mathcal{M}_{\text {inf }}$ for the lower part). The $\emptyset$ is duplicated to be present in the two parts. Naturally, $\mathcal{M}=\mathcal{M}_{\text {sup }} \cup \mathcal{M}_{\text {inf }}$. The family $\mathcal{M}_{\text {inf }}$ is clearly a family of $\mathbb{M}_{n-1}$. On the other hand, the family $\mathcal{M}_{\text {sup }}$ is a union-closed family on $U_{n}$ with the peculiarity that all its sets contain the element $n-1$ (we will denote $\mathbb{M}_{\text {sup }}$ as the set of union-closed families having this property).

Example 1. Let $\mathcal{M}$ be the family on $U_{3},\{\emptyset,\{0\},\{0,1\},\{0,2\},\{1,2\},\{0,1,2\}\}$, we can decompose this family into two closed families: $\mathcal{M}_{\text {inf }}=\{\emptyset,\{0\},\{0,1\}\}$ and $\mathcal{M}_{\text {sup }}=\{\emptyset,\{0,2\},\{1,2\},\{0,1,2\}\}$.

We will say that a $\mathbb{M}_{n-1}$ family is compatible with a $\mathbb{M}_{n}^{\text {sup }}$ family if and only if the union of the two families is closed by union. The example in the following figure illustrates that for a fixed upper part, there are several compatible lower parts.

In 14] we have shown that for a given upper family $\mathcal{M}_{\text {sup }}$, there exists a unique maximal family such that all compatible families with $\mathcal{M}_{\text {sup }}$ are the union-closed families included in the maximal family.

By way of example, the maximal family associated to $\mathcal{M}_{\text {sup }}$ (cf. figure 1 is the family $\{\emptyset,\{0\},\{1\},\{0,1\}\}$. It can be verified that the two compatible families given are in fact sub-families of this family.

The function $f: \mathbb{M}_{n}^{\text {sup }} \rightarrow \mathbb{M}_{n-1}$ defined below allows the characterization of the maximal family of an union-closed family belonging to $\mathbb{M}_{n}^{\text {sup }}$. An efficient algorithm to compute the function $f$ has been given in [14].

Definition 1. One defines the function $f: \mathbb{M}_{n}^{\text {sup }} \rightarrow \mathbb{M}_{n-1}$ such that $f(\mathcal{M})=$ $\left\{X \in 2^{U_{n-1}} \mid \forall M \in \mathcal{M}, M \cup X \in \mathcal{M}\right\}$. 


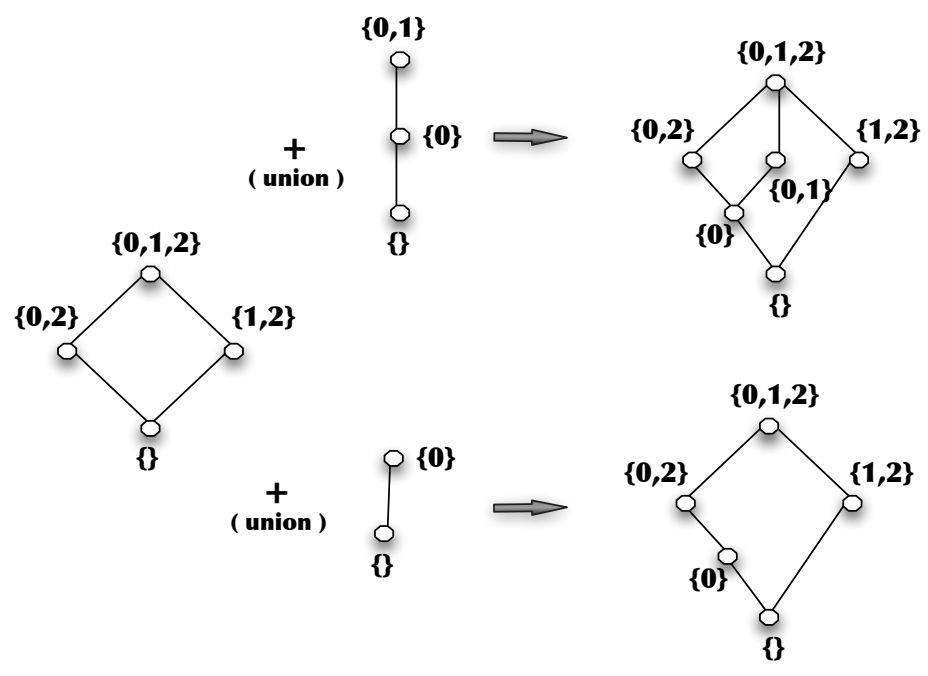

Fig. 1. On the left, a family of $\mathbb{M}_{3}^{\text {sup }}$ (all sets contain the element 2) to which are associated two different union-closed families on $U_{2}$. In the two cases the family obtained, on the right, is an union-closed family.

By the way, $f(\mathcal{M})$ represents the family of all compatible sets with $\mathcal{M}$.

Noting $\downarrow \mathcal{X}$ (for $\mathcal{X} \in \mathbb{M}_{n-1}$ ) the set of ideals of $\mathcal{X}$ in $\mathbb{M}_{n-1}$, the set of compatible families with $\mathcal{M}$ (for $\mathcal{M}$ in $\mathbb{M}_{n}^{\text {sup }}$ ) coincides with $\downarrow \mathcal{M}_{\text {max }}$, in other words $\downarrow f(\mathcal{M})$.

In fact, the set $\mathbb{M}_{n}^{\text {sup }}$ can be partitioned into two sets: the set $\mathbb{M}_{n}^{\text {sup }}$ of families that do not contain the singleton $\{n-1\}$ and the set $\mathbb{M}_{n}^{\text {sup }} p_{2}$ of families that contain it. These two sets being in natural bijection with $\mathbb{M}_{n-1}$. More formally, functions $g_{1}$ and $g_{2}$ associate with each family $\mathcal{M}$ of $\mathbb{M}_{n-1}$ a family of $\mathbb{M}_{n}^{\text {sup }_{1}}$ $\left(g_{1}(\mathcal{M})\right)$ and a family of $\mathbb{M}_{n}^{\text {sup }_{2}}\left(g_{2}(\mathcal{M})\right)$.

Definition 2. Let $g_{1}$ and $g_{2}$ be functions of $\mathbb{M}_{n-1} \rightarrow \mathbb{M}_{n}^{\text {sup }}$ such that:

- $g_{1}(\mathcal{M})=\{M \cup(n-1) \mid M \in \mathcal{M}\} \cup \emptyset \backslash\{n-1\}$

- $g_{2}(\mathcal{M})=\{M \cup(n-1) \mid M \in \mathcal{M}\} \cup \emptyset$.

See figure 2 for a graphic representation.

In fact the maximal family associated with a family of $\mathbb{M}_{n}^{s u p_{2}}$ spelt $g_{2}(\mathcal{M})$ is none other than $\mathcal{M}$. In other words for all $\mathcal{M}$ in $\mathbb{M}_{n-1}$ we have $f\left(g_{2}(\mathcal{M})\right)=\mathcal{M}$.

For the convenience of the study of the maximal family of the families of $\mathbb{M}_{n}^{\text {sup }}{ }_{1}$, we will denote $h: \mathbb{M}_{n-1} \rightarrow \mathbb{M}_{n-1}$ the function $f o g_{1}$. Thus, for all $\mathcal{M}$ of $\mathbb{M}_{n-1}, h(\mathcal{M})=f\left(g_{1}(\mathcal{M})\right)$.

From previous results and given definitions, $\mathbb{M}_{n}$ can be recursively defined as follows: 


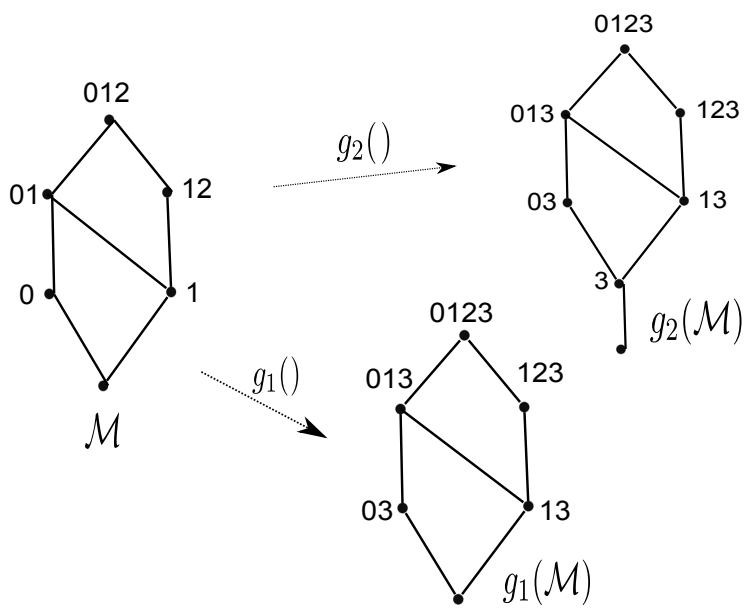

Fig. 2. On the left, a union-closed family of $\mathbb{M}_{3}$. At the bottom we find its image in $\mathbb{M}_{4}^{\text {sup }_{1}}$ by $g_{1}$. All the sets of the family contain the object 3 and the singleton $\{3\}$ doesn't belong to the family. At the top on the right, we find its image by $g_{2}$ in $\mathbb{M}_{4}^{\text {sup }}$ which contains the singleton $\{3\}$.

Theorem 1. Let $\mathbb{M}_{n}$ and $\mathbb{M}_{n-1}$ be sets of union-closed families on $U_{n}$ and $U_{n-1}$. Then:

$$
\mathbb{M}_{n}=\mathbb{M}_{n-1} \cup \bigcup_{\mathcal{M} \in \mathbb{M}_{n-1} \backslash \emptyset}\left\{g_{1}(\mathcal{M}) \times \downarrow h(\mathcal{M})\right\} \quad \cup \quad \bigcup_{\mathcal{M} \in \mathbb{M}_{n-1}}\left\{g_{2}(\mathcal{M}) \times \downarrow \mathcal{M}\right\}
$$

For interpretation and representation see figure 3 From theorem 1 we can say that there are three kinds of union-closed families on $U_{n}$ :

- the union-closed families on $U_{n-1}$, the element $n-1$ doesn't appear in any set of the family;

- the union-closed families which do not contain the singleton $\{n-1\}$. These families are under the form $g_{1}(\mathcal{M}) \cup \mathcal{M}^{\prime}$ with $\mathcal{M}$ and $\mathcal{M}^{\prime}$ in $\mathbb{M}_{n-1}$ and $\mathcal{M}^{\prime}$ included in $h(\mathcal{M})$;

- the union-closed families which contain the singleton $\{n-1\}$. These families are under the form $g_{2}(\mathcal{M}) \cup \mathcal{M}^{\prime}$ with $\mathcal{M}$ and $\mathcal{M}^{\prime}$ in $\mathbb{M}_{n-1}$ and $\mathcal{M}^{\prime}$ included in $\mathcal{M}$;

\section{About the Frankl's conjecture}

The strength of a recursive definition of an infinite set of objects is to allow planning proofs by induction of properties on this set. Nevertheless, one must 


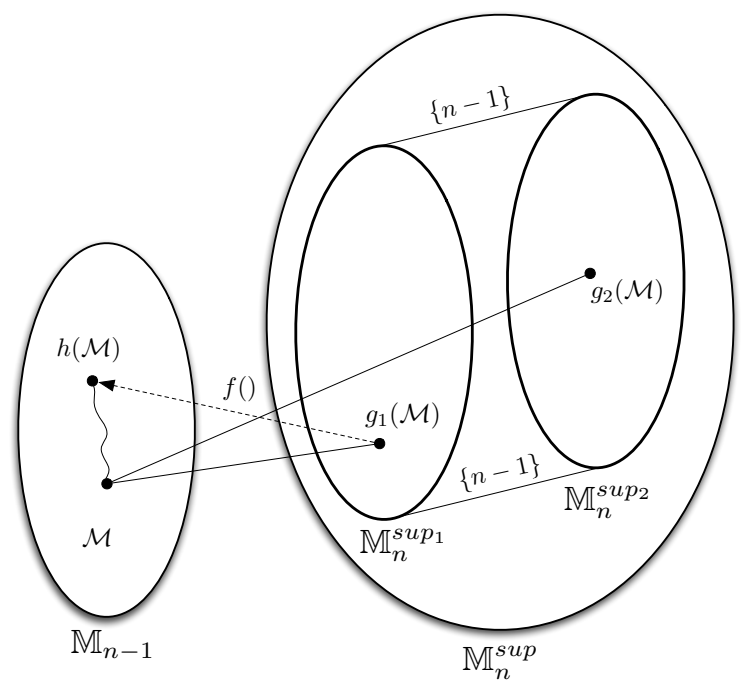

Fig. 3. On the left the order $\mathbb{M}_{n-1}$ of union-closed families on $U_{n-1}$. At the center, an isomorphic order obtained by $g_{1}$, on the right another copy obtained by $g_{2}$. Their union gives $\mathbb{M}_{n}^{\text {sup }}$. The existence of $\mathcal{M}$ in $\mathbb{M}_{n-1}$ leads to the existence of a large number of union-closed families in $\mathbb{M}_{n}$.

understand that the Frankl's conjecture concerns the structure itself of the family, rather than the set of whole numbers characterizing each set. The whole numbers are interchangeable with each other. Thus the Frankl's conjecture stays true up to a permutation. In other words, if a family is Frankl's, its image through any permutation is in itself Frankl's.

\subsection{Element of symmetry}

In our case, the principle of a demonstration by induction will fix the last whole number to be considered. But it must be possible for this role to be taken up by any one amongst them. For this reason, we will be interested in some permutations that are translations. Thus we will call $\theta$ a translation on a finite set $U_{n}=\{0, \ldots, n-1\}, \theta$ being a bijective function of $2^{U_{n}}$ in $2^{U_{n}}$. By $T_{n}$ we mean the set of $n$ translations $(a, n-1)$ for $a$ in $\{0, \ldots, n-1\}$. For a set $E \subseteq U_{n}$ and $\theta \in T_{n}$ with $\theta=(a, n-1), \theta(E)$ gives the image of $E$ by $\theta$ defined by:

- $\theta(E)=E \backslash a \cup n-1$ if $a \in E$ and $n-1 \notin E$;

- $\theta(E)=E \backslash n-1 \cup a$ if $a \notin E$ and $n-1 \in E$;

- $\theta(E)=E$ in other cases.

For any family $\mathcal{F} \subseteq 2^{U_{n}}$, by extension we will have $\theta(\mathcal{F})=\{\theta(E) \mid E \in \mathcal{F}\}$. 
Example 2. Let a family $\mathcal{F}=\{\emptyset,\{2\},\{1,2\},\{0,1,2\}\}$ and the translation $\theta=$ $(0,2)$, then $\theta(\mathcal{F})=\{\emptyset,\{0\},\{0,1\},\{0,1,2\}\}$.

Proposition 1. Let $\mathcal{F}$ be a union-closed family on $U_{n}$ and $\theta \in T_{n}$ be a translation, if $\theta(\mathcal{F})$ is a Frankl's family then $\mathcal{F}$ is Frankl's too.

\subsection{The families on $U_{n}$ containing a singleton}

One of the first results pertaining to the Frankl's conjecture was to note that any union-closed family having a singleton was "naturally" a Frankl family. This quite simple result of a conceptual viewpoint has the ability to class a large number of closed families as Frankl's family (cf. table 1). In fact, this result also appears very clearly on reading the recursive definition. By the way, for any closed family $\mathcal{F}$ containing a singleton, there is a translation $\theta$ on $U_{n}$ that sends this singleton to $\{n-1\} . \theta(\mathcal{F})$ will therefore be a family which will be written in the form $g_{2}(\mathcal{M}) \cup \mathcal{M}^{\prime}$ with $\mathcal{M}$ and $\mathcal{M}^{\prime}$ in $\mathbb{M}_{n-1}$ and $\mathcal{M}^{\prime}$ included in $\mathcal{M}$. Since, according to the definition of $g_{2}$, the sets $g_{2}(\mathcal{M})$ and $\mathcal{M}$ have same cardinal, the number of sets of $\theta(\mathcal{F})$ containing the item $n-1\left(\left|g_{2}(\mathcal{M})\right|\right)$ is greater than or equal to the number of sets not containing this item $\left(\left|\mathcal{M}^{\prime}\right|\right) \cdot \theta(\mathcal{F})$ and thereby $\mathcal{F}$ (cf. proposition 1) are therefore both Frankl's families.

\subsection{The families on $U_{n}$ not containing a singleton}

According to the recursive definition, for any closed family $\mathcal{F}$ without singleton, there exist $\mathcal{M}$ and $\mathcal{M}^{\prime}$ in $\mathbb{M}_{n-1}$ such that $\mathcal{F}$ is written as $g_{1}(\mathcal{M}) \cup \mathcal{M}^{\prime}$ with $\mathcal{M}^{\prime}$ included in $h(\mathcal{M})$. Now $h$ is an augmentation operator $(\mathcal{M} \subseteq h(\mathcal{M}))$. Thus the reasoning given further up for closed families containing the singleton $\{n-1\}$ is not valid since the part of $\mathcal{F}$ for which the sets contain the item $n-1\left(g_{1}(\mathcal{M})\right)$ is potentially of cardinal lower than its part of sets not containing the item $n-1$ $\left(\mathcal{M}^{\prime} \subseteq h(\mathcal{M})\right)$.

However, by restricting our analysis to the union-closed families $\mathcal{F}$ without singleton arising out of a family $\mathcal{M}$ in $\mathbb{M}_{n-1}$ such that $h(\mathcal{M})=\mathcal{M}$, we are assured that $\mathcal{F}$ is a Frankl family. This results in the following theorem:

Theorem 2. Let $\mathbb{C}_{n}$ be the class of union-closed families $\mathcal{F}$ on $U_{n}$ without $\{n-1\}$ such that there exist 2 families $\mathcal{M}$ and $\mathcal{M}^{\prime}$ in $\mathbb{M}_{n-1}$ with $h(\mathcal{M})=\mathcal{M}$ and $\mathcal{M}^{\prime} \subseteq \mathcal{M}$ and a translation $\theta$ in $T_{n}$ such that $\theta(\mathcal{F})$ can be written as $g_{1}(\mathcal{M}) \cup \mathcal{M}^{\prime}$ or $g_{2}(\mathcal{M}) \cup \mathcal{M}^{\prime}$. Then, families of $\mathbb{C}_{n}$ are Frankl's families.

Sketch of proof: Let $\mathcal{F}$ in $\mathbb{C}_{n}$, there exists $\theta$ in $T_{n}$ such that $\theta(\mathcal{F})=g_{1}(\mathcal{M}) \cup \mathcal{M}^{\prime}$ (the second case with $\theta(\mathcal{F})$ equal to $g_{2}(\mathcal{M}) \cup \mathcal{M}^{\prime}$ has been previously treated). The number of sets of $\theta(\mathcal{F})$ containing the element $n-1$ is $\left|g_{1}(\mathcal{M})\right|=|\mathcal{M}|$ from definition of $g_{1}$. The number of sets of $\theta(\mathcal{F})$ which do not contain $n-1$ is equal to $\left|\mathcal{M}^{\prime}\right|$ with $\mathcal{M}^{\prime}$ included in $h(\mathcal{M})$. Since, $\mathcal{M}$ is a fixed point of $h, \mathcal{M}^{\prime}$ is included in $\mathcal{M}$ and thus $\left|\mathcal{M}^{\prime}\right|$ is inferior or equal to $|\mathcal{M}| . \theta(\mathcal{F})$ as $\mathcal{F}$ are Frankl's families. See figure 4 for a graphic interpretation. 


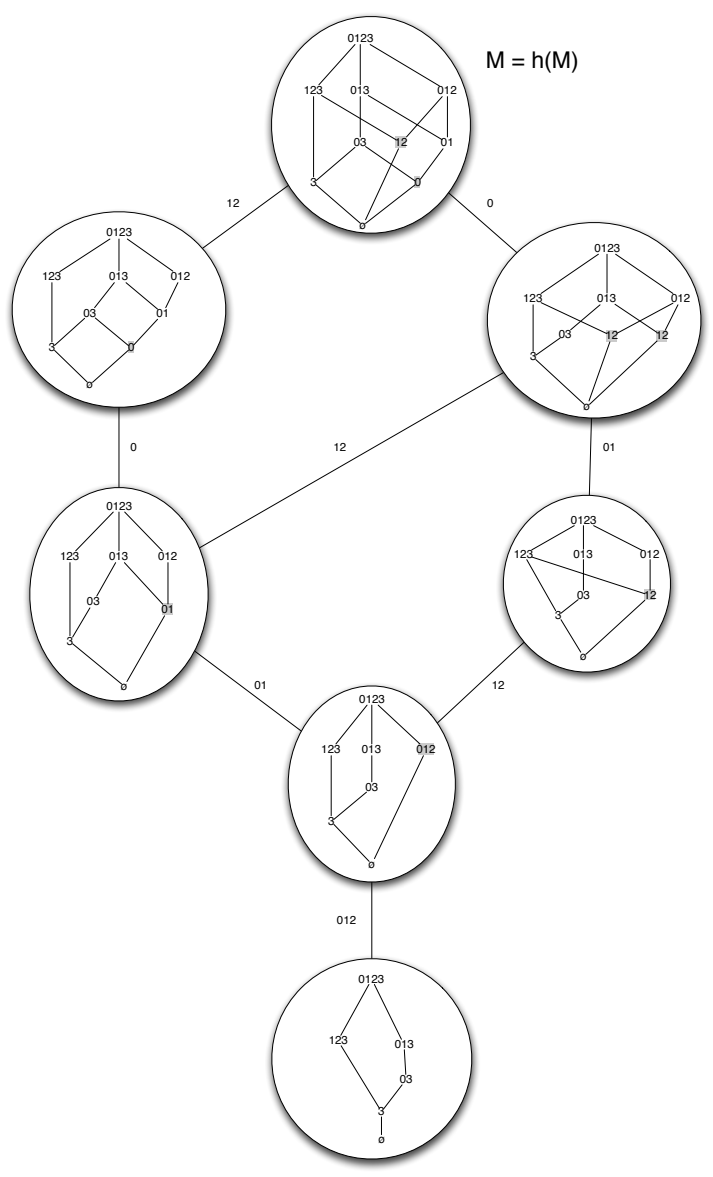

Fig. 4. Placed at the top of the order, the closed family $\mathcal{M}$ on $U_{4}$ made up of 10 sets, is a fixed point for $h$. Thus, any family $\mathcal{F}$ on $U_{5}$, written $g_{1}(\mathcal{M}) \cup \mathcal{M}^{\prime}$ for any family $\mathcal{M}^{\prime}$ given in this figure (thereby included in $h(\mathcal{M})=\mathcal{M}$ ), is a Frankl family.

Using the experience acquired to count the number of Moore families for $n=7$ (cf. [14]) we have counted the number of fixed points by $h$ for the initial values of $n$ as well as the size of the associated class. Results are presented in table 1 .

\subsection{Study of some fixed points by $h$}

Here, our intention is to show that the class $\mathbb{C}_{n}$ does not coincide with the most classes known to be Frankl's.

- Fixed point, neither modular nor distributive : The modular lattices like the distributive ones find characteristics by absence of the prohibited sub- 


\begin{tabular}{|c||c||c||c||c|}
\hline$n$ & $\left|\mathbb{M}_{n}\right|$ & $\left|\mathbb{P F}_{n}\right|$ & $\left|\mathbb{C}_{n}\right|$ & $\left|\mathbb{M}_{n}^{1}\right|$ \\
\hline 1 & 2 & 1 & & $5(83 \%)$ \\
2 & 7 & 3 & $6(85 \%)$ & $49(80 \%)$ \\
3 & 61 & 19 & $56(91 \%)$ & $2063(83 \%)$ \\
4 & 2480 & 622 & $2296(92 \%)$ & $1141766(82 \%)$ \\
5 & 1385552 & 252098 & $1250447(91 \%)$ & $140 \%$ \\
6 & 75973751474 & & $64501349128(85 \%)$ & $59424724974(78 \%)$ \\
\hline
\end{tabular}

Table 1. The first column gives the size of $\mathbb{M}_{n}$. The second column fixes the size of the set of fixed points of $\mathbb{M}_{n}$. In the third column we give the size of class $\mathbb{C}_{n}$ (we will note that the calculations of $\left|\mathbb{C}_{n}\right|$ are based on the set of fixed points of $\left.\mathbb{M}_{n-1}\right)$. Lastly, in the last column we give the number of families of $\mathbb{M}_{n}$ containing at least one singleton (noted for the occasion $\mathbb{M}_{n}^{1}$ ). For our knowledge these numbers were not known.

structure. The absence of $N_{5}$, like the "cycle" without chord with 5 vertices, characterizes the modulars and the joint absence of $N_{5}$ and the $M_{3}$ (diamond with 3 central vertices) characterizes the distributives. The family $\mathcal{M}$ given in the previous figure contains $2 N_{5}$ with the sub-families $\{\emptyset,\{0\},\{0,1\},\{1,2\},\{0,1,2\}\}$. The fixed point $\mathcal{M}$ is therefore neither modular, nor distributive. Any family generated from this fixed point will neither be modular, nor distributive.

- The presence of a $N_{5}$ in the family $\mathcal{M}$ allows us to affirm that the class $\mathbb{C}_{n}$ does not coincide either with the class defined by extension of the relatively complemented one of the lattices given in [6].

- We give with figure 5 a last example of fixed point for $h$ which does not contain singleton, nor sets of size two. The associated lattice is not modular, nor relatively complemented.

In fact there is an argument that is used to affirm that the union-closed families generated from fixed points do not have any specific property. Let $\mathcal{B}$ be the Boolean lattice in $U_{n-1}, \mathcal{B}$ is a fixed point by $h$. Moreover, since any closed family in $U_{n-1}$ is included in $\mathcal{B}$, for any $\mathcal{M}$ in $\mathbb{M}_{n-1}, \mathcal{M} \cup g_{1}(\mathcal{B})$ belongs to $\mathbb{C}_{n}$. Thus the union-closed family $\mathcal{M} \cup g_{1}(\mathcal{B})$ cannot be characterized by any property other than those shared by the set of union-closed families of which the upper part is boolean (for example the families whose upper part is Boolean contain as the smallest set, 2-element sets).

\section{Conclusion}

In this article we defined a new class $\mathbb{C}_{n}$ of families for which the Frankl's conjecture is true. This class represents more than $85 \%$ of the union-closed families for the initial values of $n$. However, we will note that the class $\mathbb{C}_{n}$ contains the families having a singleton and that the number of these families is itself large. The definition of $\mathbb{C}_{n}$ is based on the recursive definition of the set of union-closed families and on an argument in particular that allows classifying 


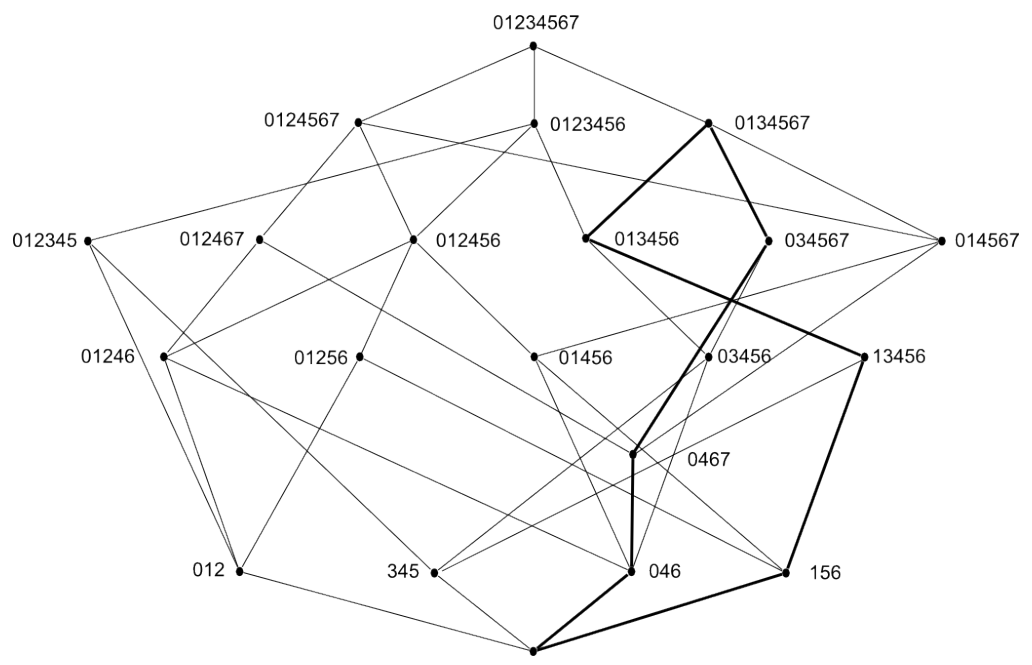

Fig. 5. A fixed point by $h$ on $U_{8}$ which doesn't contain singleton nor 2-element set. The associated lattice to this family is not distributif, modular, or relatively complemented. Last, it doesn't contain a bloc as defined by Poonen in [6].

as "Frankl's" families having a singleton. In some ways the class $\mathbb{C}_{n}$ contains families $\mathcal{F}$ arising from a family of $\mathbb{M}_{n-1}$ stable by the operator $f\left(g_{i}()\right)$ for $i=1$ or $i=2$ (up to a translation).

It seems that until now the study of the Frankl's conjecture consisted of determining the classes of families for which the conjecture was verified. We think that with the help of the recursive definition, the approach could now be different and should consist of understanding why the families that are not in $\mathbb{C}_{n}$ are "Frankl's". While naturally supposing that the conjecture is true.

\section{References}

1. On Graphs, N.A.S.I., Order, Rival, I., Organization., N.A.T. Graphs and order: the role of graphs in the theory of ordered sets and its applications / edited by Ivan Rival. D. Reidel Pub. Co.; Sold and distributed in the U.S.A. and Canada by Kluwer Academic Publishers, Dordrecht, Holland; Boston: Hingham, MA, U.S.A.(1985)

2. D. G. Sarvate, J.C.R.: On the union-closed sets conjecture. Ars Combin. 27 149-153 (1989)

3. Frankl, P.: Extremal set systems. 1293-1329 (1995)

4. Stanley, R.P.: Enumerative Combinatorics, Vol I. The Wadsworth and Brooks Cole Mathematics Series (1986)

5. Abe, T.: Strong semimodular lattices and frankl's conjecture. Algebra Universalis $44(2000)$

6. Poonen, B.: Union-closed families. J. Comb. Theory Ser. A 59(2) 253-268 (1992)

7. Abe, T., Nakano, B.: Frankl s conjecture is true for modular lattices. Graphs and Combinatorics 14 305-311 (1998) 
8. Abe, T., Nakano, B.: Lower semimodular types of lattices: Frankl s conjecture holds for lower quasi-semimodular lattices. Graphs and Combinatorics 16 1-16 (2000)

9. Ivica Bosnjak, P.M.: The 11-element case of frankl's conjecture. The electronic journal of combinatorics $\mathbf{1 5}$ (2008)

10. C', P.M. An attempt at frankl s conjecture (2007)

11. Johnson, R.T., Vaughan, T.P.: On union-closed families, i. J. Comb. Theory Ser. A 84(2) 242-249 (1998)

12. Morris, R.: Fc-families and improved bounds for frankl s conjecture. Eur. J. Comb. 27(2) 269-282 (2006)

13. Vaughan, T.P.: Families implying the frankl conjecture. Eur. J. Comb. 23(7) 851-860 (2002)

14. Colomb, P., Irlande, A., Raynaud, O.: Counting of moore families on $n=7$. In: ICFCA, LNAI 5986. (2010) 